\title{
SAR Image Enhancement Based On Fractional Fourier Transform
}

\author{
Tan Gewei*, Pan Guangwu and Lin Wei
}

School of Information Science and Engineering, Huaqiao University, Xiamen, Fujian, 361021, P.R. China

\begin{abstract}
Fractional Fourier transform (FrFT) is a kind of generalized Fourier transform and linear frequency modulation signals can be well focused after FrFT. Meanwhile motion error is an important factor affecting SAR (synthetic aperture radar) resolution, aiming at the problem that the effect of error elimination is not obvious in processing non-stationary motion error using the conventional SAR imaging algorithm, FrFT is combined with two-step motion compensation technique and wavenumber domain imaging algorithm in this paper. Simulation results and imaging results of real SAR data show that the proposed algorithm indeed eliminates the influence of motion error effectively. (the real SAR data provided by Institute of Electronics, Chinese Academy of Sciences).
\end{abstract}

Keywords: Fractional Fourier transform, motion error, two-step motion compensation, wavenumber algorithm, SAR resolution

\section{INTRODUCTION}

The system theory and related technology for SAR are established on keeping the flight path of radar platform in a straight line, but the atmospheric turbulence or other natural factors often make the aircraft deviated from the nominal track and generating motion error of antenna phase center, which will cause the amplitude modulation and phase modulation of radar echo signals, result in the image blurring and geometric distortions [1-3].

Fractional Fourier transform (FrFT) is proposed by V.Namias in 1980 [4], which is a new time-frequency analysis tool. Compared to Fourier transform, FrFT has incomparable superiority in processing non-stationary signals and linear frequency modulation signals. Linear frequency modulation signals can be well focused after fractional Fourier transform with the specific rotation angle, which provides a possibility to achieve high resolution and high accuracy in SAR imaging [5-7].

There have been literatures putting forward SAR imaging algorithms combining with fractional Fourier transform. Literature [8] proposed an improving CS imaging algorithm based on FrFT. Literature [9] also proposed a combination of FrFT and RD imaging algorithm. These research results show that FrFT can contribute to the improvement of SAR resolution.

In order to eliminate the influence of motion error more effectively and to improve SAR resolution, FrFT is combined with two-step motion compensation technology and SAR imaging algorithms in the paper, through utilizing the superiority of FrFT in processing chirp signals and nonstationary signals, so as to obtain high quality SAR images.

\section{FRACTIONAL FOURIER TRANSFORM}

Fractional Fourier transform and its inverse transform for signal $x(t)$ is defined as.

$$
\begin{aligned}
& X_{p}(u)=F_{p}(x)=\int_{-\infty}^{\infty} x(t) K_{p}(t, u) d t \\
& x(t)=F_{-p}(X)=\int_{-\infty}^{\infty} X_{p}(u) K_{-p}(t, u) d u
\end{aligned}
$$

where,

$$
K_{p}(t, u)=\left\{\begin{array}{lc}
\sqrt{\frac{1-j \cot \alpha}{2 \pi}} \exp \left\{j \pi\left(\left(t^{2}+u^{2}\right) \cot \alpha-2 u t \csc \alpha\right)\right\} & \alpha \notin n \pi \\
\delta(t-u) & \alpha \in n \cdot 2 \pi \\
\delta(t+u) & \alpha+\pi \in n \cdot 2 \pi
\end{array}\right.
$$

and $p=\frac{2}{\pi} \alpha$ which is the order of FrFT. When $p=1$, FrFT is Fourier transform.

Fractional Fourier transform for linear frequency modulation signal $x(t)=e^{j 2 \pi k t^{2}}$ is as follow:

$$
\begin{aligned}
X_{p}(u)=F_{p}(x) & =\int_{-\infty}^{\infty} x(t) K_{p}(t, u) d t \\
& =A e^{j \pi u^{2} \cot \alpha} \int_{-\infty}^{\infty} e^{j 2 \pi k t^{2}} e^{j \pi t^{2} \cot \alpha-j 2 \pi u t \csc \alpha} d t
\end{aligned}
$$

where $A=\sqrt{\frac{1-j \cot \alpha}{2 \pi}}$, when $\cot \alpha=-2 k, p=-\frac{2}{\pi} \operatorname{arccot}(2 k) \quad p_{\text {opt }}$ is the optimal order of FrFT. Thus,

$$
\begin{aligned}
X(u) & =F_{p}\left[e^{j 2 \pi k t^{2}}\right]=A e^{-j 2 \pi k u^{2}} \int_{-\infty}^{\infty} e^{-j 2 \pi u t \csc \alpha} d t \\
& =2 \pi A e^{-j 2 \pi k u^{2}} \delta(2 \pi \csc \alpha u)=\frac{A}{\csc \alpha} \delta(u)=C \delta(u)
\end{aligned}
$$




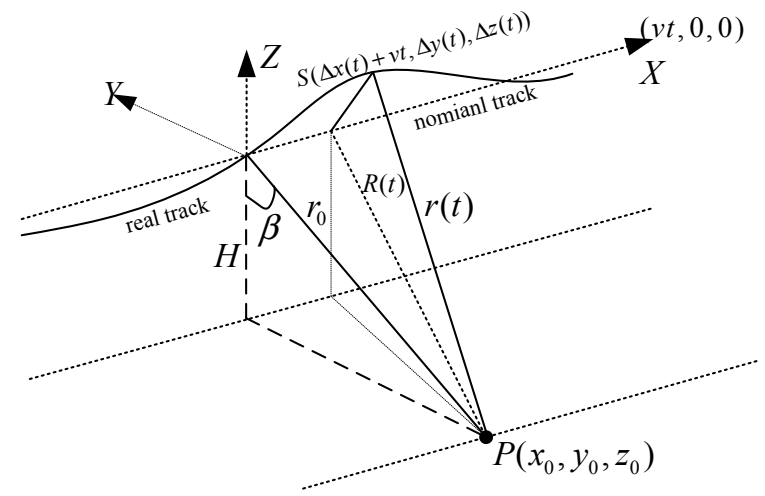

Fig. (1). Geometry model of SAR system under the positive side mode.

Formula (3) shows the optimal order FrFT for a chirp signal is a impulse signal. Just using the optimal-order fractional Fourier transform, chirp signals will be well focused, which no needs additional matching compression, thereby improving the processing efficiency.

\section{MOTION ERROR MODEL AND SAR ECHO SIG- NALS}

Motion error refers to the difference of the distance from one target to real track and the distance from the target to the nominal track. By taking the target $P$ in Fig. (1), for example, the deviation from the nominal flight path is $(\Delta x(t), \Delta y(t), \Delta z(t))$, the real distance is $r(t)$ and the nominal distance is $R(t)$. The slant range with motion error is as follow.

$$
\begin{aligned}
& r(t)=\sqrt{\left[\Delta x(t)+v t-x_{0}\right]^{2}+\left[\Delta y(t)-y_{0}\right]^{2}+\left[\Delta z(t)-z_{0}\right]^{2}} \\
& =R(t)+\Delta r_{0}(t)+\Delta r_{a v}(t)
\end{aligned}
$$

where $r_{0}=\sqrt{y_{0}^{2}+z_{0}^{2}}, y_{0}=r_{0} \sin \beta, z_{0}=r_{0} \cos \beta, R(t)=\sqrt{r_{0}^{2}+\left(v t-x_{0}\right)^{2}}$.

$$
\Delta r_{0}(t)=\Delta y(t) \sin \beta+\Delta z(t) \cos \beta=\Delta r_{r e f}\left(t, r_{r e f}\right)+\Delta r_{r v}\left(t, r_{0}\right)
$$

which is motion error in range direction and can be divided as range-independent error $\Delta r_{r e f}$ and range-dependent error $\Delta r_{r v}[10]$.

$\Delta r_{a v}$ is motion error in azimuth direction, the influence of motion error to different point target in azimuth direction as shown in Fig. (2).

When azimuth motion error is $\Delta x$, the change of slant range for target $P$ is

$$
\Delta r_{P}=P S-P S_{0} \approx \frac{(\Delta x)^{2}}{2 r_{0}}
$$

and that for target $P_{l}$ is

$$
\Delta r_{p_{1}}=P_{1} S-P_{1} S_{0}=\sqrt{r_{0}^{2}+\left(x_{L}-\Delta x\right)^{2}}-\sqrt{r_{0}^{2}+x_{L}^{2}} \approx-\frac{\left(2 x_{L}-\Delta x\right) \Delta x}{2 r_{0}}
$$

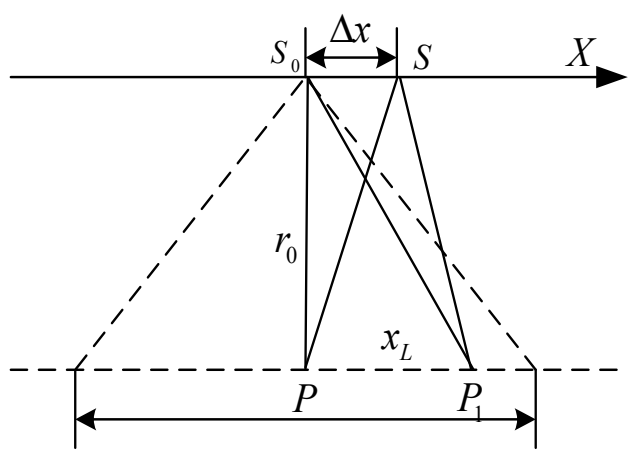

Fig. (2). Motion error for different point target in azimuth direction.

where $x_{L}$ is half of the synthetic aperture length, when azimuth beam is wide, the azimuth space-variant properties of motion error is obvious.

Suppose that SAR transmit the chirps to an observed scene, their echoes are

$$
\begin{aligned}
s d\left(t, \tau ; r_{0}\right)= & \sigma\left(x_{0}, r_{0}\right) \cdot \operatorname{rect}\left(\frac{\tau-2 r / c}{T_{p}}\right) \exp \left\{-j \pi k\left(\tau-\frac{2 r}{c}\right)^{2}\right\} \\
& \cdot \operatorname{rect}\left(\frac{t-t_{0}}{T_{s}}\right) \exp \left\{-j \frac{4 \pi r}{\lambda}\right\}
\end{aligned}
$$

where $\tau$ is fast time in the slant range direction, $t$ is slow time along the radar flight path, $c$ and $\lambda$ are the speed of light and the radar wavelength respectively, and $k$ is the chirp rate. $\operatorname{rect}()$ is the rectangle function, in which $T_{p}$ and $T_{s}$ are the pulse duration and synthetic aperture time. $r(t)$ is the instant range from the radar to a point target in the observed scene, shown as Eq. (4).

Ignoring azimuth motion error, Fourier transform in range direction first is performed by stationary phase principle and the result is [11] as follow:

$$
\begin{aligned}
s D\left(t, f_{r} ; r_{0}\right)=C_{1} \cdot \sigma\left(x_{0}, r_{0}\right) \cdot \operatorname{rect}\left(\frac{f_{r}}{B_{r}}\right) \cdot \operatorname{rect}\left(\frac{t-t_{0}}{T_{s}}\right) \cdot \exp \left\{j \pi \frac{f_{r}^{2}}{k}\right\} \\
\cdot \exp \left\{-j \frac{4 \pi r_{0}}{c} f_{r}\right\} \cdot \exp \left\{-j \frac{4 \pi \Delta r_{r e f}\left(t, r_{r e f}\right)}{c} f_{r}\right\} \\
\cdot \exp \left\{-j \frac{4 \pi r_{0}}{\lambda}\right\} \cdot \exp \left\{-j \frac{4 \pi \Delta r_{r e f}\left(t, r_{r e f}\right)}{\lambda}\right\} \cdot \exp \left\{-j \varphi_{r v}\left(t ; r_{0}\right)\right\}
\end{aligned}
$$

\section{TWO-STEP MOTION COMPENSATION TECH COMBINED WAVENUMBER DOMAIN ALGO- RITHM BASED ON FRFT}

Stolt interpolation is a key step in conventional two-step motion compensation combined wavenumber domain algorithm, which not only increases the amount of calculation, but also brings additional error. Fractional Fourier transform can replace the interpolation operation and can transform signals into range-Doppler domain at the same time, thus improving the calculation efficiency and imaging resolution. 


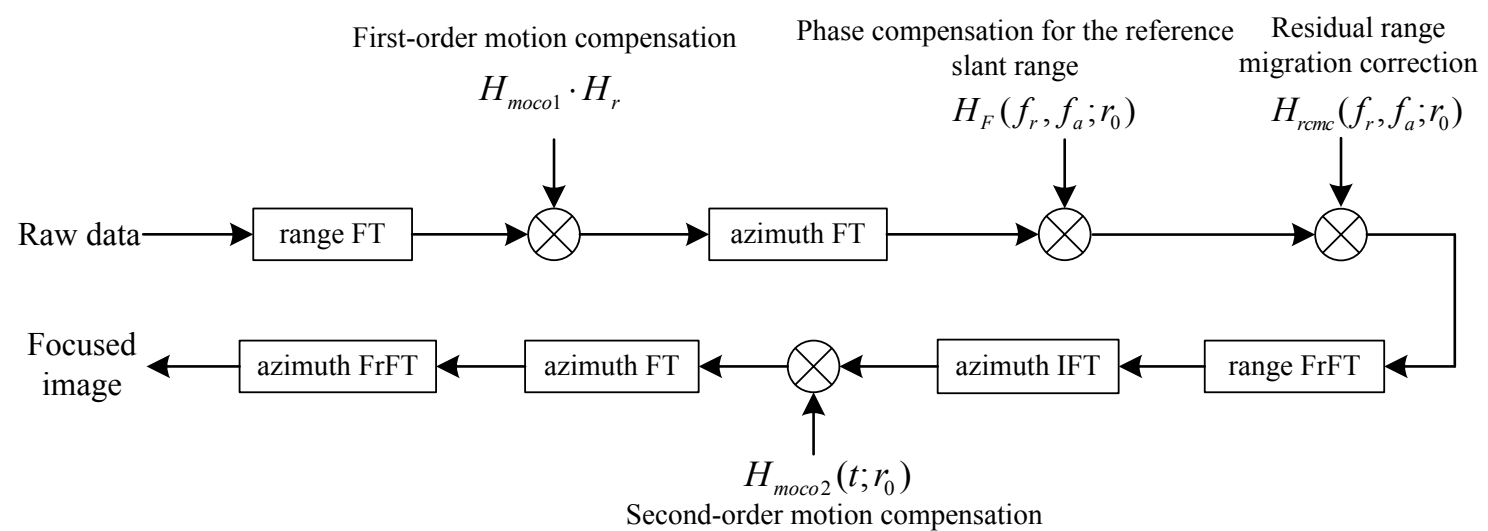

Fig. (3). Processing flow for the two-step motion compensation combined wavenumber domain algorithm based on FrFT.

Fig. (3) is processing flow of the two-step motion compensation combined wavenumber domain algorithm based on FrFT.

First Performing Fourier transform on the range to the raw data (shown as Eq. 7), then multiplying $H_{\text {mocol }}$ and $H_{r}$ to complete range focusing and first-order motion compensation. The corresponding compensation functions are

$H_{\text {mocol }} \cdot H_{r}=\exp \left\{j \frac{4 \pi \Delta r_{r e f}\left(t, r_{r e f}\right)}{c}\left(f_{r}+f_{c}\right)\right\} \exp \left\{-\pi \frac{f_{r}^{2}}{K_{r}}\right\}$

Next performing Fourier transform in azimuth direction, the processed signal is inverted into two-dimensional frequency domain and the result is

$$
\begin{aligned}
S D\left(f_{a}, f_{r} ; r_{0}\right)= & C_{2} \cdot \sigma \cdot r e c t\left[\frac{f_{r}}{B_{r}}\right] \cdot \exp \left\{j \psi\left(f_{a}, f_{r} ; r_{0}\right)\right\} \\
& \cdot \operatorname{rect}\left[\frac{f_{a}}{B_{d}}\right] \cdot \exp \left\{-j \varphi_{r v}\left(f_{a} ; r_{0}\right)\right\}
\end{aligned}
$$

where $\varphi_{r v}$ is phase error caused by the range-variant motion error. $\psi\left(f_{a}, f_{r} ; r_{0}\right)=-\frac{4 \pi r_{0}}{\lambda} \sqrt{\left(1+\frac{f_{r}}{f_{c}}\right)^{2}-\left(\frac{\lambda f_{a}}{2 v}\right)^{2}}$, which can be divided into two parts according the reference range,

$\psi\left(f_{a}, f_{r} ; r_{0}\right)=\psi_{0}\left(f_{a}, f_{r} ; r_{m}\right)+\psi_{1}\left(f_{a}, f_{r} ; r_{0}\right)$

$\psi_{0}\left(f_{a}, f_{r} ; r_{m}\right)=-\frac{4 \pi r_{m}}{\lambda} \sqrt{\left(1+\frac{f_{r}}{f_{c}}\right)^{2}-\left(\frac{\lambda f_{a}}{2 v}\right)^{2}}$

$\psi_{1}\left(f_{a}, f_{r} ; r_{0}\right)=-\frac{4 \pi \delta r}{\lambda} \sqrt{\left(1+\frac{f_{r}}{f_{c}}\right)^{2}-\left(\frac{\lambda f_{a}}{2 v}\right)^{2}}$

where $r_{0}=r_{m}+\delta r, \delta r \in\left[-w_{r} / 2, w_{r} / 2\right], r_{m}$ is slant range of the swath band center, $w_{r}$ is the width of swath band.

$\psi_{0}\left(f_{a}, f_{r} ; r_{m}\right)=-\frac{4 \pi r_{m}}{c}\left[\begin{array}{l}f_{c} \gamma\left(f_{a}\right)+\frac{f_{r}}{\gamma\left(f_{a}\right)}-\frac{1-\gamma\left(f_{a}\right)^{2}}{2 f_{c} \gamma\left(f_{a}\right)^{3}} f_{r}^{2} \\ +\frac{1-\gamma\left(f_{a}\right)^{2}}{2 f_{c}^{2} \gamma\left(f_{a}\right)^{5}} f_{r}^{3}+\cdots\end{array}\right]$
The first item corresponds to azimuth compression, second to range cell migration, third to second range compression, the fourth is high-order coupling item of range and azimuth. So choosing the following compensation function,

$H_{F}\left(f_{a}, f_{r} ; r_{m}\right)=\exp \left\{-j \psi_{0}\left(f_{a}, f_{r} ; r_{m}\right)+\frac{4 \pi r_{m}}{\lambda} \sqrt{1-\left(\frac{\lambda f_{a}}{2 v}\right)^{2}}\right\}$

Multiplying (9) with $H_{F}$ can completing range cell migration correction, second range compression and phase compensation for high-order coupling item of range and azimuth for the reference range.The signal after this step is

$$
\begin{aligned}
& S D_{\delta r}\left(f_{a}, f_{r} ; r_{0}\right)= C_{2} \cdot \sigma \cdot r e c t\left[\frac{f_{r}}{B_{r}}\right] \cdot \operatorname{rect}\left[\frac{f_{a}}{B_{d}}\right] \\
& \cdot \exp \left\{j \psi_{2}\left(f_{a}, f_{r} ; r_{0}\right)\right\} \cdot \exp \left\{-j \varphi_{r v}\right\} \\
& \psi_{2}\left(f_{a}, f_{r} ; r_{0}\right)=-\frac{4 \pi r_{0}}{\lambda} \gamma\left(f_{a}\right)-\frac{4 \pi \delta r}{c}\left[\begin{array}{l}
\frac{f_{r}}{\gamma\left(f_{a}\right)}-\frac{1-\gamma\left(f_{a}\right)^{2}}{2 f_{c} \gamma\left(f_{a}\right)^{3}} f_{r}^{2} \\
+\frac{1-\gamma\left(f_{a}\right)^{2}}{2 f_{c}^{2} \gamma\left(f_{a}\right)^{5}} f_{r}^{3}+\cdots
\end{array}\right]
\end{aligned}
$$

then, multiplying $H_{r c m c}=\exp \left\{j \frac{4 \pi \delta r}{c} \frac{f_{r}}{\gamma\left(f_{a}\right)}\right\}$, and performing fractional Fourier transform to invert the processed signal into range-Doppler domain,

$$
\begin{aligned}
& S d_{1}\left(f_{a}, \tau ; r_{0}\right)=\int_{-\infty}^{+\infty}\left[S D_{\delta r}\left(f_{a}, f_{r} ; r_{0}\right) \cdot H_{r c m c}\left(f_{r}, f_{a} ; r_{0}\right)\right] K_{p}\left(f_{r}, \tau\right) d f_{r} \\
& =C_{3} \cdot \operatorname{rect}\left[\frac{f_{a}}{B_{d}}\right] \int_{-\infty}^{+\infty} \operatorname{rect}\left[\frac{f_{r}}{B_{r}}\right] \exp \left\{j \psi_{2}\left(f_{a}, f_{r} ; r_{0}\right)\right\} \\
& \cdot \exp \left\{j \pi\left(\left(f_{r}^{2}+\tau^{2}\right) \cot \alpha-2 f_{r} \tau \csc \alpha\right)\right\} d f_{r} \\
& =C_{4} \cdot \sigma \cdot r e c t\left[\frac{f_{a}}{B_{d}}\right] \cdot \exp \left\{-j \frac{4 \pi r_{0}}{\lambda} \gamma\left(f_{a}\right)\right\} \cdot \exp \left\{-j \varphi_{r v}\right\} \cdot \sin c\left(\tau-\frac{2 r_{0}}{c}\right)
\end{aligned}
$$

where $\alpha=-\operatorname{arccot}\left(\frac{2 \delta r}{c} \cdot \frac{1-\gamma\left(f_{a}\right)^{2}}{f_{c} \gamma\left(f_{a}\right)^{3}}\right)$, the optimal order of

FrFT is $p_{\text {opt }}=\frac{2}{\pi} \alpha$. 
Table 1. Simulations parameters.

\begin{tabular}{|c|c|}
\hline Parameter & value \\
\hline \hline Carrier frequency & $1.5 \mathrm{GHz}$ \\
\hline Bandwidth for transmit signal & $150 \mathrm{MHz}$ \\
\hline Pulse duration of transmit signal & $1.5 \mu \mathrm{s}$ \\
\hline The number of range sampling points & 1024 \\
\hline Forward velocity & $180 \mathrm{~m} / \mathrm{s}$ \\
\hline Length of synthetic aperture & $320 \mathrm{~m}$ \\
\hline The number of azimuth sampling points & 512 \\
\hline Range-invariant motion error & $t^{2}$ \\
\hline
\end{tabular}

Next, performing azimuth Fourier transform into twodimensional time-domain, implementing second-order motion compensation, the corresponding phase compensation function is

$$
H_{\text {moco } 2}\left(t, r_{0}\right)=\exp \left\{j\left(\varphi_{\text {mo }}\left(t, r_{0}\right)-\frac{4 \pi}{\lambda} \Delta r_{r e f}\left(t, r_{r e f}\right)\right)\right\}
$$

Finally, in range-Doppler domain, completing azimuth compression by using FrFT and get focused image. The result is,

$$
\begin{aligned}
& \begin{array}{l}
s d\left(t, \tau ; r_{0}\right)=\int_{-\infty}^{+\infty} S d_{2}\left(f_{a}, \tau ; r_{0}\right) \cdot K_{p}\left(f_{a}, t\right) d f_{a} \\
=C_{5} \cdot \sigma \cdot \sin c\left(\tau-\frac{2 r_{0}}{c}\right) \int_{-\infty}^{+\infty} \operatorname{rect}\left[\frac{f_{a}}{B_{d}}\right] \cdot \exp \left\{-j \frac{4 \pi r_{0}}{\lambda} \gamma\left(f_{a}\right)\right\} \\
\cdot \quad \exp \left\{j \pi\left(\left(f_{a}^{2}+t^{2}\right) \cot \beta-2 f_{a} t \csc \beta\right)\right\} d f_{a}
\end{array} \\
& =C_{6} \cdot \sigma \cdot \sin c\left(\frac{t}{\sin \beta}\right) \cdot \sin c\left(\tau-\frac{2 r_{0}}{c}\right) \\
& \text { where } \gamma\left(f_{a}\right)=\sqrt{1-\left(\frac{\lambda f_{a}}{2 v}\right)^{2}} \approx 1-\frac{1}{2}\left(\frac{\lambda f_{a}}{2 v}\right)^{2} \text { and } \beta=-\cot ^{-1}\left(\frac{\lambda r_{0}}{2 v^{2}}\right), \text { the opti- } \\
& \text { mal order of FrFT is } p_{\text {opt }}=\frac{2}{\pi} \beta .
\end{aligned}
$$

\section{SIMULATIONS AND SAR IMAGING RESULTS BASED ON REAL DATA FOR THE PROPOSED AL- GORITHM}

\subsection{Simulation Results to Point Target with Motion Er- ror in the Range Direction for the Algorithm}

Simulation results for the proposed algorithm are as follow, the simulation parameters as shown in Table $\mathbf{1}$.

The range error is non-stationary random motion error whose mean value is exponential function, so range-variant motion error is $\Delta r_{r v}\left(t, r_{0}\right)=\Delta r_{0}\left(t, r_{0}\right)-\Delta r_{r e f}\left(t, r_{r e f}\right)$.

Simulations to point target with motion error using FFT based the two-step motion compensation combined wave- number domain algorithm and FrFT based the two-step motion compensation combined wavenumber domain algorithm are shown in Fig. (4), (a) is comparison of azimuth impulse response with no error and after motion compensation using traditional algorithm; (b) is comparison of azimuth impulse response with no error and after motion compensation using the proposed algorithm; (c) is comparison of range impulse response after motion compensation by the two algorithm; (d) is comparison of azimuth impulse response after motion compensation by the two algorithm.

Fig. (4b) shows that the main lobe of impulse response in azimuth direction is narrower and the influence of quadratic phase error is eliminated virtually. The wider mainlobe of Fig. (4a) indicates the influence of quadratic phase error still exists, which proves the processing effect of non-stationary motion error using FrFT is obvious than FFT, at the same time proves that the focusing effect of chirp signal using FrFT is better than FFT. The performance comparison of their impulse response in the azimuth is shown in Table 2.

\subsection{Imaging Results Comparison for the Proposed Algo- rithm and the Conventional Algorithm}

In Fig. (5), (a) is the imaging result of the real SAR data with motion errors, the blurring image shows there are obvious quadratic phase errors. Processing result for such SAR data using the conventional FFT based two step motion compensation wavenumber domain algorithm is shown in (b), due to the elimination of most motion errors, image resolution is improving significantly, but in some places with more details (such as part of the circle line), the image is not clear and image resolution deteriorates because of the residual phase error. The processing result with the proposed FrFT based two step motion compensation wavenumber domain algorithm is shown in (c), details information increases and the image resolution is further improving.

\section{THE REALIZATION OF CERAMIC DESIGN SYS- TEM}

Motion error is a crucial factor to limit airborne SAR resolution improving. The two-step motion compensation combined wavenumber domain algorithm based on fractional 


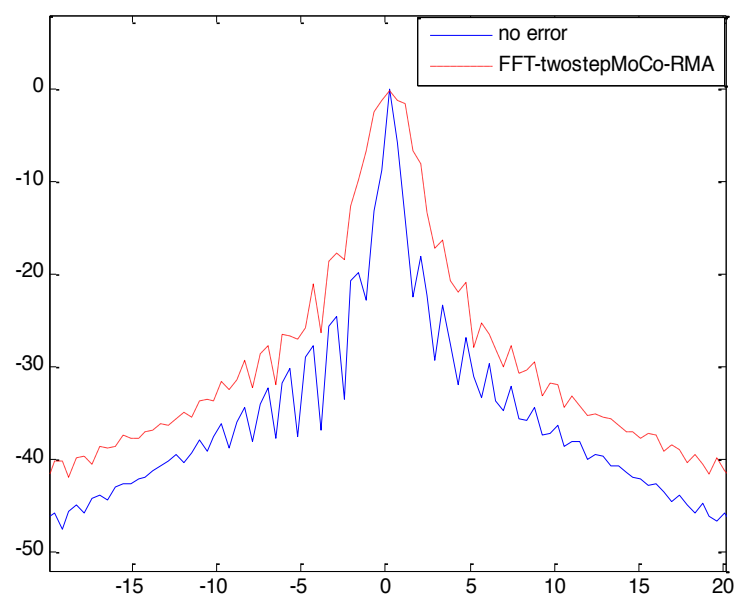

(a)

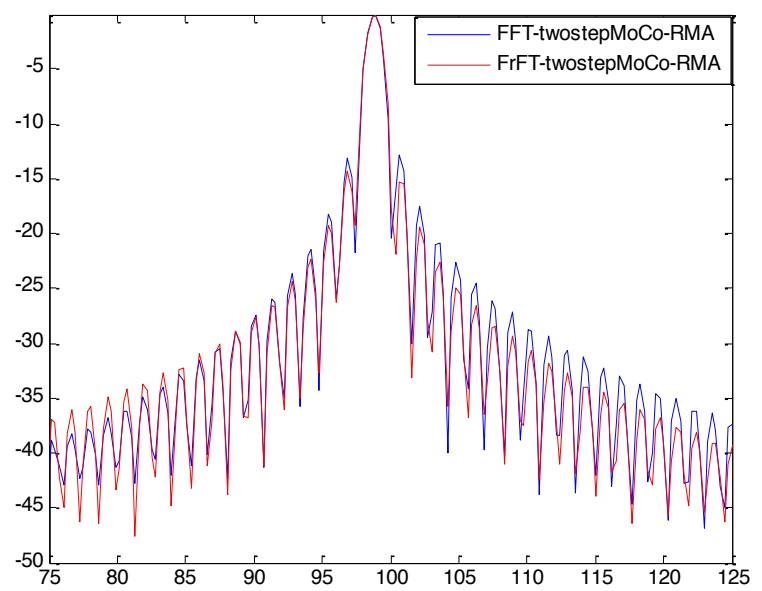

(c)

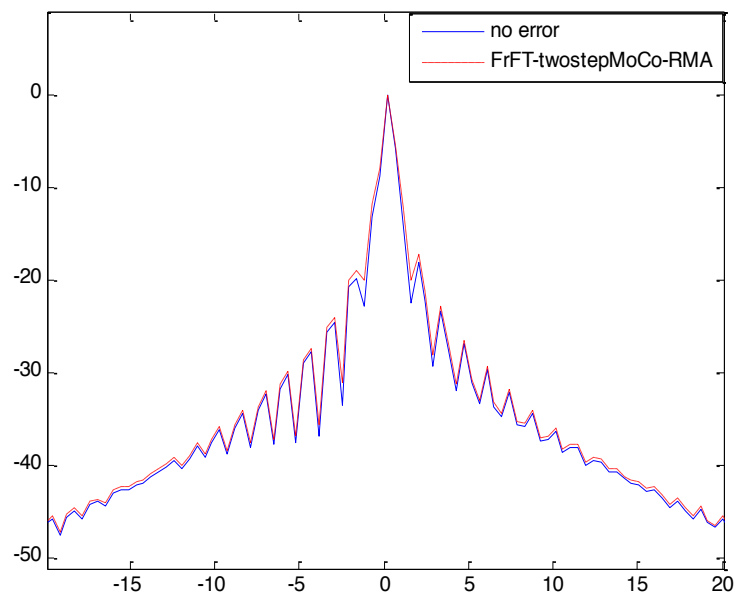

(b)

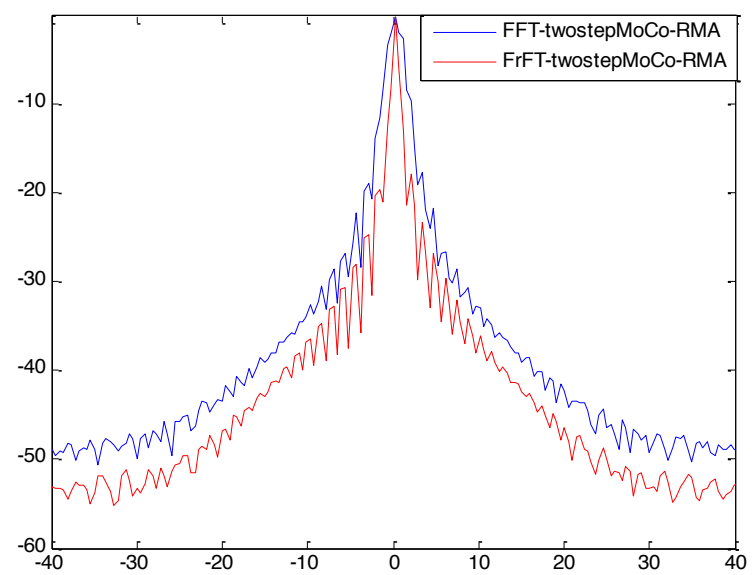

(d)

Fig. (4). The teapot three-dimensional model material library.

Table 2. Performance comparison of impulse response in azimuth direction.

\begin{tabular}{|c|c|c|c|}
\hline Motion Compensation Algorithm & The Wide of Main Lobe & Integrated Side Lobe Ratio & Peak to Side Lobe Ratio \\
\hline \hline No error & $1.10 \mathrm{~m}$ & $-26.012 \mathrm{~dB}$ & $-31.432 \mathrm{~dB}$ \\
\hline FFT-twostep motion compensation RMA & $4.864 \mathrm{~m}$ & $-24.681 \mathrm{~dB}$ & $-24.218 \mathrm{~dB}$ \\
\hline FrFT-twostep motion compensation RMA & $1.125 \mathrm{~m}$ & $-25.589 \mathrm{~dB}$ & $-30.353 \mathrm{~dB}$ \\
\hline
\end{tabular}

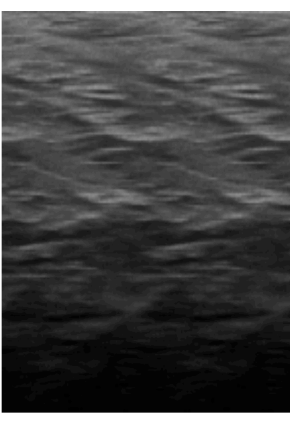

(a)

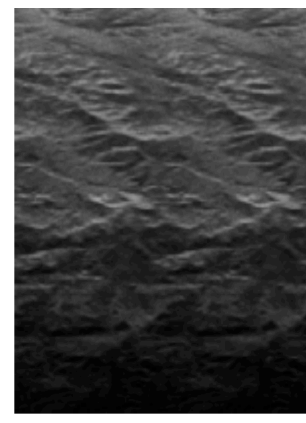

(b)

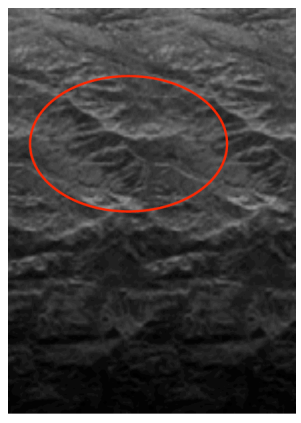

(c)

Fig. (5). The teapot three-dimensional model material library. 
Fourier transform proposed in this paper can solve various motion errors effectively, especially can eliminate the image blurring caused by non-stationary motion errors, the research provides an effective solution scheme for motion error.

\section{CONFLICT OF INTEREST}

The author confirms that this article content has no conflict of interest.

\section{ACKNOWLEDGEMENTS}

This paper is supported by the natural science foundation of Fujian province of 2013 project (the number is 2013J01242).

\section{REFERENCES}

[1] Y. Li, X. Liang, C. Ding, L. Zhou, L. Chen, W. Hong, "A Motion Compensation Approach Integrated in the Omega-K Algorithm for Airborne SAR", IEEE Int. Conf. Imaging Syst. Tech., Manchester, United Kingdom, 2012, pp. 245-248.

[2] Y. Mao, M. Xiang, L. Wei, Y. Li and W. Hong, "Error Analysis of SAR Motion Compensation", IEEE Int. Conf. Imaging Syst. Tech., Manchester, United Kingdom, 2012, pp. 377-380.
[3] H. Guo, Y. Li, "Studying Atmospheric Turbulence Effects on Aircraft Motion for Airborne SAR Motion Compensation Requirements", IEEE International Conference on Imaging Systems and Techniques, Manchester, United Kingdom, 2012, pp. 152-157.

[4] V. Namias, "The Fractional Fourier Transform and Its Application in Quantum Mechanics", J. Inst. Math, vol. 25, pp. 241-165, 1980.

[5] Q. lin, T. Ran, Z. Siyong, W. Yue, "Adaptive Time-Varying Filter for Linear FM Signal in Fractional Fourier Domain", $6^{\text {th }}$ IEEE Int. Conf. Signal Process., Beijing, China, 2002, pp.1425-1428.

[6] L. Durak, S. Aldirmaz, "Adaptive Fractional Fourier Domain Filtering”, Signal Processing, vol. 90, no. 4, pp. 1188-1196, 2010.

[7] S. Ervin, D. Igor, S. LJubisa, "Fractional fourier transform as a signal processing tool: an overview of recent developments", Signal Process., vol. 91, no. 6, pp. 1351-1369, 2011.

[8] A. S. Amein, "A new chirp scaling algorithm based on the fractional fourier transform", IEEE Signal. Process. Lett., vol. 12, no. 10, pp. 705-708, 2005.

[9] C. Clemente, J. J. Soraghan, "Range Doppler and chirp scaling processing of synthetic aperture radar data using the fractional Fourier transform", IET Signal. Process., vol. 6, no. 5, pp. 503-510, 2012.

[10] R. Lanari, G. Fornaro, "A short discussion on the exact compensation of the SAR range-dependent range cell migration effect", IEEE Trans. Geosci. Remote Sens., vol. 35, pp. 1446-1452, 1997.

[11] G. Tan, Y. Deng, "The two-dimensional spatial-variant properties of airborne SAR motion error and its compensation", J. Electron. Inform. Tech., vol. 31, no. 2, pp. 366-369, 2009.

Received: September 07, 2014

(C) Gewei et al.; Licensee Bentham Open.

This is an open access article licensed under the terms of the Creative Commons Attribution Non-Commercial License (http://creativecommons.org/licenses/by-nc/3.0/) which permits unrestricted, non-commercial use, distribution and reproduction in any medium, provided the work is properly cited. 